Review

\title{
Superconductivity in Layered Organic Metals
}

\section{Jochen Wosnitza}

Hochfeld-Magnetlabor Dresden, Helmholtz-Zentrum Dresden-Rossendorf, 01314 Dresden, Germany; E-Mail: j.wosnitza@hzdr.de; Tel.: +49-351-260-3527; Fax: +49-351-260-3531.

Received: 6 March 2012; in revised form: 14 April 2012 / Accepted: 16 April 2012 /

Published: 19 April 2012

\begin{abstract}
In this short review, I will give an overview on the current understanding of the superconductivity in quasi-two-dimensional organic metals. Thereby, I will focus on charge-transfer salts based on bis(ethylenedithio)tetrathiafulvalene (BEDT-TTF or ET for short). In these materials, strong electronic correlations are clearly evident, resulting in unique phase diagrams. The layered crystallographic structure leads to highly anisotropic electronic as well as superconducting properties. The corresponding very high orbital critical field for in-plane magnetic-field alignment allows for the occurrence of the Fulde-FerrellLarkin-Ovchinnikov state as evidenced by thermodynamic measurements. The experimental picture on the nature of the superconducting state is still controversial with evidence both for unconventional as well as for BCS-like superconductivity.
\end{abstract}

Keywords: organic superconductors; electronic structure; superconducting state; Fulde-Ferrell-Larkin-Ovchinnikov state

\section{Introduction}

More than 30 years ago the first organic superconductor, (TMTSF) $)_{2} \mathrm{PF}_{6}$, was discovered by D. Jérome et al. [1]. Materials based on TMTSF (tetramethyltetraselenafulvalene) with a monovalent anion are electronically quasi-one-dimensional (1D) materials that show very rich pressure-temperaturemagnetic field phase diagrams with formidable ground-state properties. For some time a possible triplet superconducting state was discussed, but recent experimental data prove the singlet nature with presumed $d$-wave symmetry. More information on these topical issues can be found in a number of reviews [2-4].

Here, I want to focus on the quasi-two-dimensional (2D) organic superconductors. Among these, materials with BEDT-TTF (a.k.a. ET, bis(ethylenedithio)tetrathiafulvalene) as organic building block 
(Figure 1, left side) are the most investigated class with superconducting transition temperatures, $T_{c}$, of up to about $13 \mathrm{~K}[2,4,5]$. The most common stoichiometry of these $2 \mathrm{D}$ organic metals is (BEDT-TTF $)_{2} X$, where $X$ represents a monovalent anion such as $\mathrm{I}_{3}^{-}, \mathrm{Cu}(\mathrm{NCS})_{2}^{-}, \mathrm{Cu}\left[\mathrm{N}(\mathrm{CN})_{2}\right] \mathrm{Br}^{-}$, or $\mathrm{SF}_{5} \mathrm{CH}_{2} \mathrm{CF}_{2} \mathrm{SO}_{3}^{-}$. Charge transfer from the neutral BEDT-TTF molecule to the anion leads to partially filled molecular bands, resulting in metallic conductivity. Apart from BEDT-TTF, the organic molecule BETS (bisethylenedithio-tetraselenafulvalene), where the inner four sulfur atoms of BEDT-TTF are replaced by selenium, can be utilized as building block for a remarkable class of organic superconductors [6]. For example, charge-transfer salts containing $\mathrm{Fe}^{3+}$ anions may lead to competing magnetic and superconducting interactions, reentrant superconductivity upon cooling, and magnetic-field-induced superconductivity.

Figure 1. (left) The molecular structure of bis(ethylenedithio)tetrathiafulvalene (BEDT-TTF or ET); (right) Schematic arrangement of the ET and anion layers in the quasi-two-dimensional organic metals.

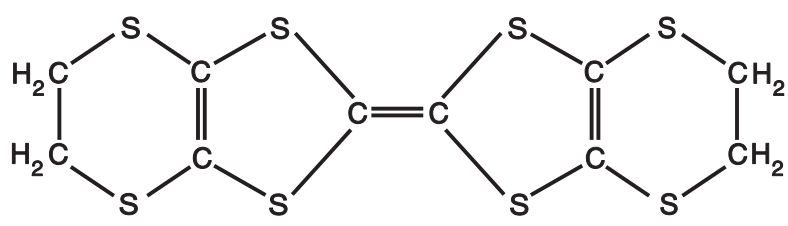

anion

layer

"insulating"

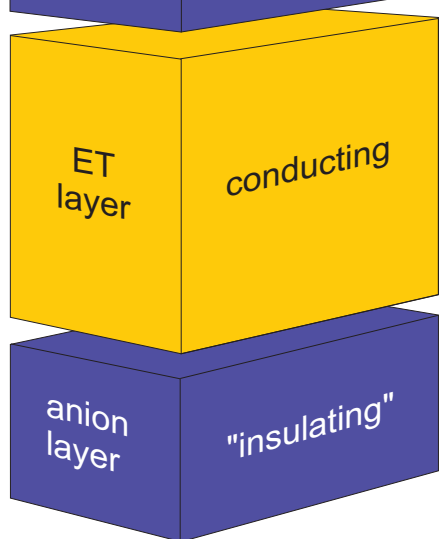

Besides superconductivity, the organic metals show a large variety of different ground states such as antiferromagnetism, spin-Peierls, spin-density-wave, and charge-density-wave states. These states are accessible by tuning the structure, counter anion, magnetic field, temperature, and pressure. The study of these fertile phase diagrams has led to new theoretical concepts; however, a solid understanding of some of these states still remains a challenge. This includes the normal metallic state which often appears to be not explicable by conventional Fermi-liquid theory.

Many superconducting properties of the 2D organic superconductors, such as the $H-T$ phase diagram and the vortex dynamics, resemble the behavior found in the cuprate high- $T_{c}$ superconductors [7-11]. In contrast to the latter, however, much less is known about the nature of the superconducting state in organic metals. Clear-cut experimental proof for unconventional non-BCS-like superconductivity, such as phase-sensitive experimental evidence, is missing. For the organic superconductors, where singlet pairing has been established, the experimental picture on the symmetry of the order parameter is still diverse (see [4,5,12-15] for recent reviews). Some results hint at line nodes in the superconducting 
energy gap, i.e., $d$-wave symmetry, whereas other experiments, in particular, specific-heat data, suggest the existence of a nodeless energy gap, fully consistent with BCS-like $s$-wave symmetry.

A further unsolved question is the mechanism responsible for the Cooper-pair coupling. While there is some belief in the existence of a non-phonon-mediated coupling mechanism, as appears to be operative in unconventional superconductors such as the cuprates, $\mathrm{Sr}_{2} \mathrm{RuO}_{4}$, and heavy-fermion compounds, this issue remains unclear and heavily debated for the organic superconductors. The proximity of antiferromagnetic or spin-density-wave states to the superconducting state spurred the notion that antiferromagnetic fluctuations are responsible for the coupling. On the other hand, a number of experiments prove the existence of clear interrelations between phonons and superconductivity.

\section{Crystallographic and Electronic Structure}

Mostly, the term "organic materials" implies biological substances or plastics, i.e., polymeric carbon-based chains. However, the organic metals discussed here are charge-transfer salts of mostly high crystalline quality. They usually are grown by electrocrystallization from appropriate solutions, the details of which are reviewed in depth in Reference [16]. During this process the oxidation of the donor molecules occurs together with the crystal growth at inert electrodes. The 2D organic charge-transfer salts typically grow as single-crystalline plates that weigh up to a few milligrams.

A number of different crystal structures exists for the ET-based charge-transfer salts. They mainly differ in the packing motifs of the ET molecules and are labeled by different Greek letters [2,16]. The common feature, however, is the layered crystal structure depicted in the right panel of Figure 1. In the conducting ET layers, there are sufficiently close contacts between the $\pi$ orbitals of the sulfur atoms of neighboring ET molecules. This leads to 2D molecular bands with rather small in-plane anisotropies (typically within a factor of two). The anions, sandwiched between the highly conducting ET layers, are responsible for the charge transfer that leads to partially filled molecular bands, enabling metallic conductivity. The electrical resistivity perpendicular to the layers is larger by about 3-6 orders of magnitude than for electronic transport within the ET layers. One should note, however, that the exact resistive anisotropy is difficult to determine since any dislocation disturbing the in-plane transport will immediately add a large out-of-plane component to the measured resistance $[4,14]$.

Although the layered structure occurs in all ET-based superconductors, the varying in-plane packing motifs of the organic molecules lead to different 2D electronic structures. By utilizing the rather simple extended Hückel tight-binding approximation, the in-plane electronic band structures can be calculated. Four examples of the resulting energy dispersions and of the in-plane Fermi surfaces are shown in Figure 2. These calculations, in most cases, agree extraordinarily well with the experimentally determined Fermi surfaces, although the large number of atoms per unit cell necessitates quite crude simplifications in the tight-binding approximations. More sophisticated band-structure calculations either led to incorrect Fermi-surface topologies due to oversimplification or finally confirmed all in all the Hückel results [17]. It is remarkable that in spite of the complex crystallographic structure the electronic structure is astonishingly simple. Indeed, the in-plane Fermi surfaces often resemble free-electron topologies. 
Figure 2. Calculated band structures and Fermi surfaces for (a) $\beta$-phase (after Reference [18]), (b) $\kappa$-phase [21,22], (c) $\alpha$-phase [23], and (d) $\beta^{\prime \prime}$-phase BEDT-TTF salts [25].
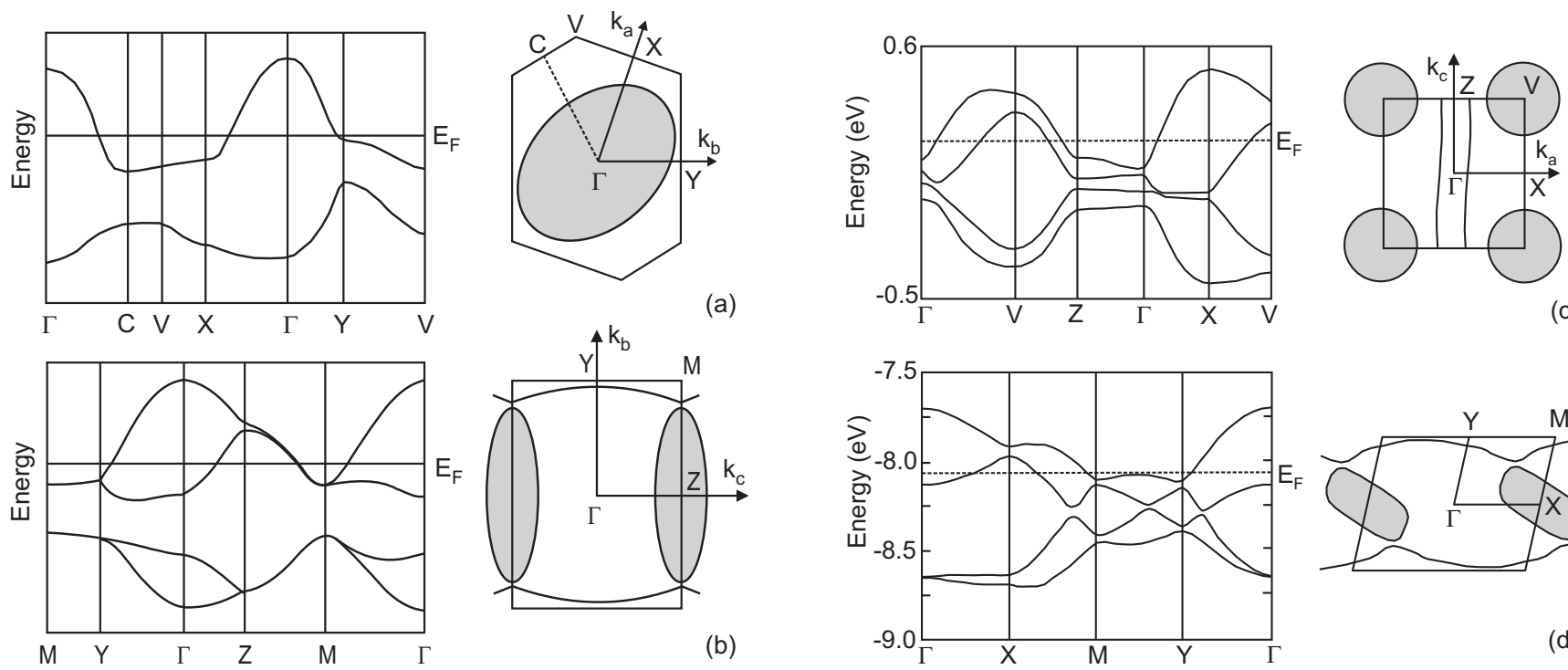

(c)

(b)

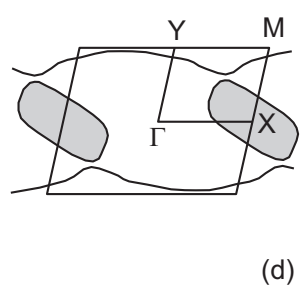

For the $\beta$ phase, one electron per unit cell is transferred to the anion leaving the highest occupied molecular orbital half filled. The nearly isotropic in-plane overlap integrals lead to an almost circular free-electron-like Fermi surface (Figure 2a) [18]. The validity of this result has been verified by measurements of magnetic quantum oscillations and angular-dependent magnetoresistance oscillations [17,19]. Indeed, only one closed orbit with the predicted area (shaded area in Figure 2a) and only slightly more cornered shape $[19,20]$ could be reconstructed from the data.

The Fermi surfaces of the other crystalline phases with pairs of dimerized ET molecules are almost as simple as for the $\beta$ phase. The dimerization doubles the unit cell, resulting in two transferred electrons on two molecular orbitals crossing the Fermi surface as visualized in Figure $2 b-d$. The resulting Fermi surfaces again resemble the free-electron picture. Since the dimerization is halving the first Brillouin zone, the almost free-electron-like Fermi-surface ellipse cuts the first Brillouin zone. Consequently, gaps open at the zone boundary due to Bragg reflections. These gaps are smaller for the $\kappa$-phase materials [21,22], but larger for the $\alpha$-phase salts. Besides the shaded closed orbits, wavy open bands appear (Figure 2c) [23]. These 1D bands are prone to a density-wave transition which indeed is realized in some of the $\alpha$-phase organic salts [24].

Although for most organic metals the calculated Fermi surfaces are favorable in line with experimental data, the measured Fermi surface in $\beta^{\prime \prime}$-(BEDT-TTF) $)_{2} \mathrm{SF}_{5} \mathrm{CH}_{2} \mathrm{CF}_{2} \mathrm{SO}_{3}$ differs noticeably from the prediction (Figure 2d) [25]. The closed orbits are found to be only about $1 / 3$ of the calculated orbits and so far no direct evidence for the open bands has been reported. Another drawback of the extended Hückel band-structure calculations is the negligence of many-body effects such as electron-electron and electron-phonon interactions. Consequently, the calculated effective masses are often much smaller than the measured values. 


\section{Properties of the Superconducting State}

Before discussing the still controversial issues on the nature of the superconducting state in the organic metals, I will present some principal superconducting properties of the $2 \mathrm{D}$ organic metals. These properties resemble in many respect the behavior observed also in the cuprate superconductors. The 2D organic metals are strongly type-II superconductors with lower critical fields, $\mu_{0} H_{c 1}$, of only a few $\mathrm{mT}$ and upper critical fields, $\mu_{0} H_{c 2}$, in the Tesla range. These fields depend strongly on the direction of the applied field. For $H$ applied within the ET planes, $H_{c 2}$ often exceeds the Pauli paramagnetic limit [26,27] and evidence for the Fulde-Ferrell-Larkin-Ovchinnikov state may be found, as will be discussed below. The moderately low upper critical fields allow, in contrast to the cuprate high- $T_{\mathrm{c}}$ superconductors, to access conveniently the whole superconducting phase diagram and to study the rich physics of the vortex phase [4,5]. In the following, some selected experimental features observed for the organic superconductors are presented.

\subsection{Principal Properties}

As a typical example, Figure 3 shows the superconducting phase diagram of the well-studied material $\kappa$-(BEDT-TTF $)_{2} \mathrm{Cu}\left[\mathrm{N}(\mathrm{CN})_{2}\right] \mathrm{Br}$ for fields applied perpendicular to the ET planes. Shown are the temperature dependences of $\mu_{0} H_{c 1}, \mu_{0} H_{c 2}$, and of the irreversibility field, $\mu_{0} H_{i r r}$ derived from DC-magnetization, $M_{d c}$, and AC-susceptibility, $\chi_{a c}$, measurements [28-30]. The upper critical fields (closed circles) were obtained from a scaling analysis of the magnetization data which due to superconducting fluctuations are strongly rounded in fields [28]. The lower critical fields (closed diamonds) were extracted from the vanishing magnetic relaxation, i.e., the fields where no longer a time dependence of the magnetization is detected [29]. From Figure 3, the very large range of the Shubnikov state, characteristic for strongly type-II superconductors, is evident (note the logarithmic scale). The extrapolated critical fields at $T=0$ are $(12 \pm 2) \mathrm{T}$ for the upper critical field and $(3 \pm 1) \mathrm{mT}$ for the lower critical field [28,29]. This results in a thermodynamic critical field of about $100 \mathrm{mT}$, an in-plane coherence length $\xi_{\|} \approx 5 \mathrm{~nm}$, and a Ginzburg-Landau parameter of $\kappa \approx 100$.

For fields parallel to the ET layers, $\mu_{0} H_{c 1}$ is considerably smaller than the earth's field and a reliable determination of the lower critical fields is challenging [31]. From the initial slope of the in-plane uppercritical-field line close to $T_{c}$, an orbital critical field far beyond $100 \mathrm{~T}$ may be estimated (see below). This results in an interplane coherence length much smaller than the layer distance of about $1.6 \mathrm{~nm}$ [7]. Therefore, in order to establish a three-dimensional phase coherence, the superconducting layers are Josephson coupled. That indeed has been verified directly by measuring Josephson plasma resonances in magneto-optical experiments [32]. Superconductivity in the "insulating" or badly-conducting anion layers is induced by phase-coherent Cooper pairs in the ET layers. Above a threshold field, $\mu_{0} H_{i r r}$, for this proximity-induced superconductivity, the layers are effectively decoupled, and the vortices are weakly pinned $2 \mathrm{D}$ objects (pancake vortices). This can be realized from the temperature dependence of the irreversibility field, $\mu_{0} H_{i r r}$. A distinct crossover from $3 \mathrm{D}$ vortex fluctuations close to $T_{\mathrm{c}}$ to $2 \mathrm{D}$ fluctuations at lower temperatures is observed (Figure 3) [29]. 
Figure 3. Temperature dependences of the lower $\left(H_{c 1}\right)$ and upper $\left(H_{c 2}\right)$ critical fields as well as the irreversibility fields $\left(H_{i r r}\right)$ determined by different techniques for fields applied perpendicular to the ET layers. The data for $H_{c 2}$ (closed circles) and $H_{i r r}$ (open triangles) are taken from [28].

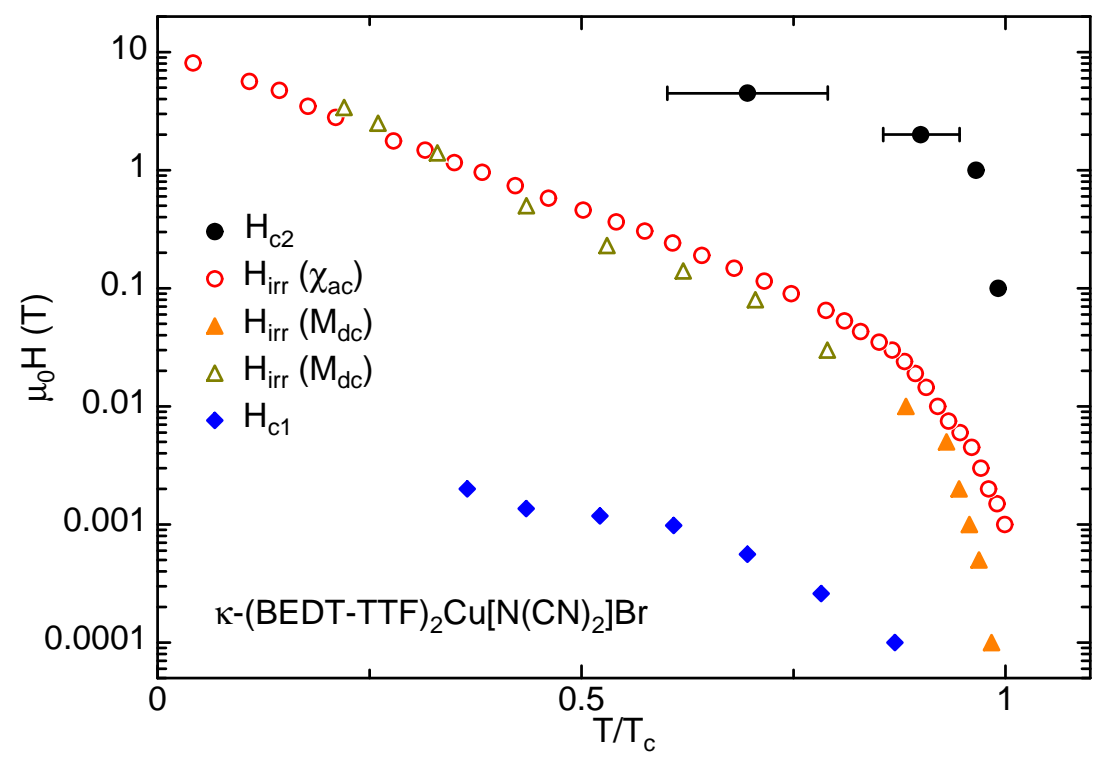

As mentioned, for the 2D organic metals, the destruction of superconductivity due to orbital effects appears at extraordinarily high in-plane magnetic fields. In that case, however, spin effects become relevant. For singlet superconductors, when the Zeeman energy becomes larger than the condensation energy, the Cooper pairs finally break up. This occurs at the Pauli-paramagnetic limit, $\mu_{0} H_{P}=\Delta_{0} /\left(\sqrt{2} \mu_{B}\right)$, with $\mu_{B}$ the Bohr magneton and $\Delta_{0}$ the superconducting energy gap at $T=0$ [27]. However, even beyond this limit superconductivity can survive in special states where the superconducting order parameter is spatially modulated. The existence of such states were predicted independently by Fulde and Ferrell (FF) [33] as well as Larkin and Ovchinnikov (LO) in 1964 [34].

In the FFLO state, Cooper pairs can only exist with a finite center-of-mass momentum due to the Zeeman-split Fermi surfaces. This results in an oscillating part of the order parameter in real space with a wavelength of the order of the coherence length. Two conditions are necessary for the FFLO state to occur. First, the so-called Maki parameter, $\alpha=\sqrt{2} H_{\text {orb }} / H_{P}$ [35], should be larger than 1.8 [36] and, second, the superconductor needs to be in the clean limit with a mean-free path much larger than the coherence length.

These conditions are favorably fulfilled in many 2D organic superconductors. Mostly, these materials are clean-limit superconductors and, when the magnetic field is oriented exactly parallel to the ET layers, the orbital critical field becomes much larger than the Pauli limit, as mentioned above. Indeed, in a number of works, indications for the existence of the FFLO state have been reported for some of the 2D organic metals [37]. The experimental evidence, however, was mostly based on transport measurements. Only recently, thermodynamic data allowed to conclude the existence of the FFLO state [38].

For $\kappa$-(BEDT-TTF $)_{2} \mathrm{Cu}(\mathrm{NCS})_{2}$, specific-heat measurements in magnetic fields aligned parallel to the 2D layers showed spike-like anomalies for fields above $21 \mathrm{~T}$ and temperatures below $3 \mathrm{~K}$. This can be seen clearly in Figure 4, where the specific-heat difference, $\Delta C$, divided by temperature as a function of 
temperature is plotted. For that, the normal-state specific heat, measured at $14 \mathrm{~T}$ applied perpendicular to the layers, has been subtracted for each curve [38]. At about $21 \mathrm{~T}$, the specific-heat anomaly at the superconducting transition sharpens and at $21.5 \mathrm{~T}$ a spike in $\Delta C$ occurs. This is evidence for a first-order nature of the phase transition. For higher fields, a second very sharp anomaly appears within the superconducting state. This proves the existence of an additional thermodynamic phase. The latter anomaly shows a well-resolvable hysteresis, while the superconducting transition at higher temperatures has a smaller hysteresis (see [38] for details).

Figure 4. (a) Temperature dependence of the specific-heat difference between the superconducting and normal state, $\Delta C / T$, of $\kappa$-(BEDT-TTF $)_{2} \mathrm{Cu}(\mathrm{NCS})_{2}$ in magnetic fields applied parallel to the superconducting layers; (b) High-field data in an expanded scale.
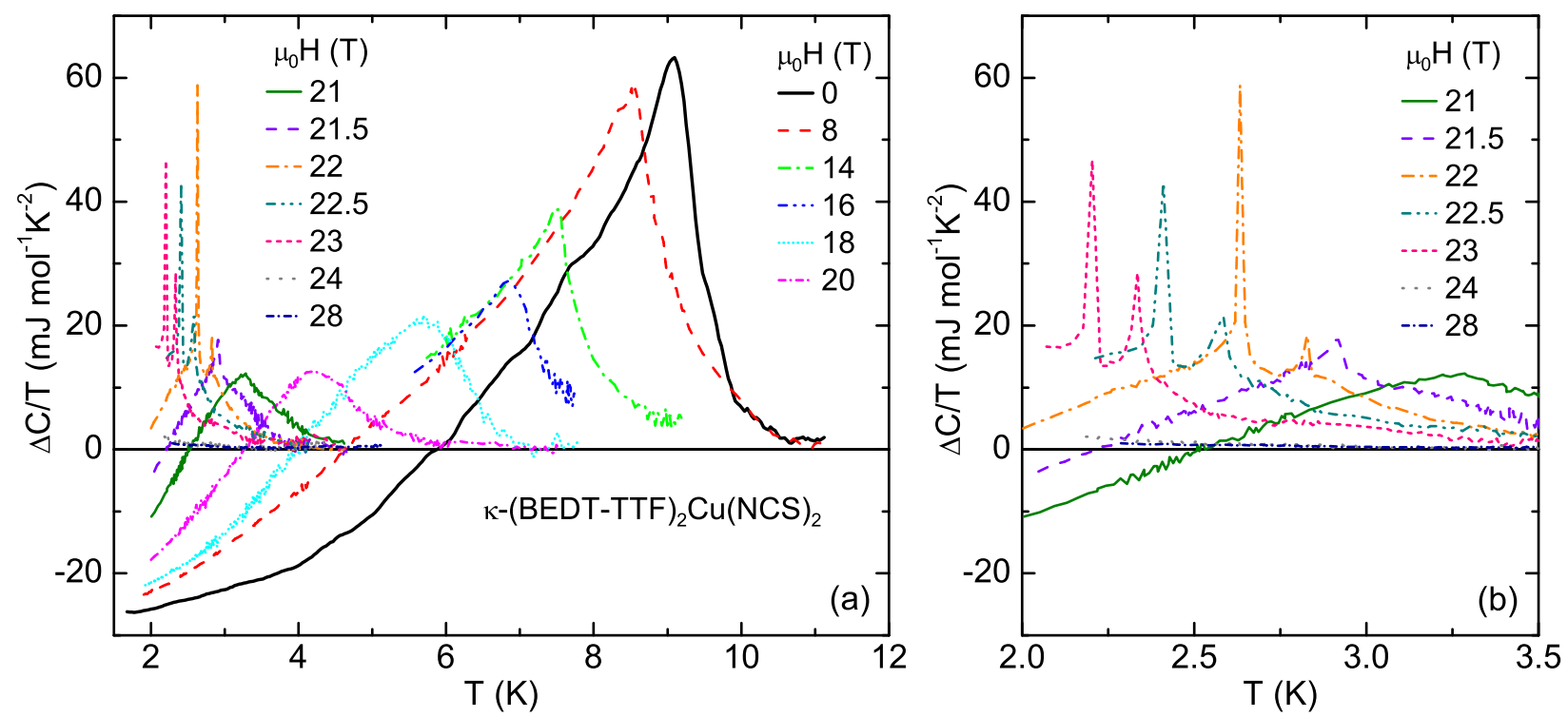

Further evidence for the existence of the FFLO state has been gathered from magnetic-torque studies (Figure 5a) [39]. Upon lowering the temperature, the magnetic torque, which is proportional to the magnetization, shows an increasingly sharp transition at $\mu_{0} H_{c 2}$. This indicates that the Pauli limit, $\mu_{0} H_{P}$, is reached. Anyhow, for even lower temperatures superconductivity survives even beyond this field. In addition, a second dip-like feature appears in the torque signal below $\mu_{0} H_{c 2}$ (vertical arrows in Figure 5), signaling the emergence of the FFLO phase. These results nicely confirm the specific-heat data for the $\mu_{0} H_{c 2}$ line. There is, however, a discrepancy in the extension of the FFLO phase. Whereas for the specific heat the second anomaly suggests a very narrow region for the additional thermodynamic phase, the dip-like feature in the torque data stays almost constant at about the Pauli limit. This can be explained by the much better in-plane alignment of the magnetic field in the torque measurements made possible by an in situ rotation of the torque platform. In the specific-heat experiment for $\kappa$-(BEDT$\mathrm{TTF})_{2} \mathrm{Cu}(\mathrm{NCS})_{2}$ no rotation of the sample platform was possible. Indeed, recent angular-dependent specific-heat measurements of the organic superconductor $\beta^{\prime \prime}$-(BEDT-TTF) ${ }_{2} \mathrm{SF}_{5} \mathrm{CH}_{2} \mathrm{CF}_{2} \mathrm{SO}_{3}$ supported this notion. These latter data showed that the FFLO state disappears for out-of-plane orientations of the magnetic field as small as $0.5^{\circ}$. A second specific-heat anomaly appeared only for field angles between about $0.2^{\circ}$ and $0.3^{\circ}$. 
Figure 5. (a) Magnetic-torque data of $\kappa$-(BEDT-TTF $)_{2} \mathrm{Cu}(\mathrm{NCS})_{2}$ at various temperatures for in-plane magnetic fields aligned along the $b$ direction. For $T=1.7 \mathrm{~K}$, data for both up and down field sweeps, otherwise only down sweeps are shown. The vertical arrows mark the small dip-like features, which are associated with the transition into the FFLO state;

(b) Schematic phase diagram deduced from the data shown in (a) [39].
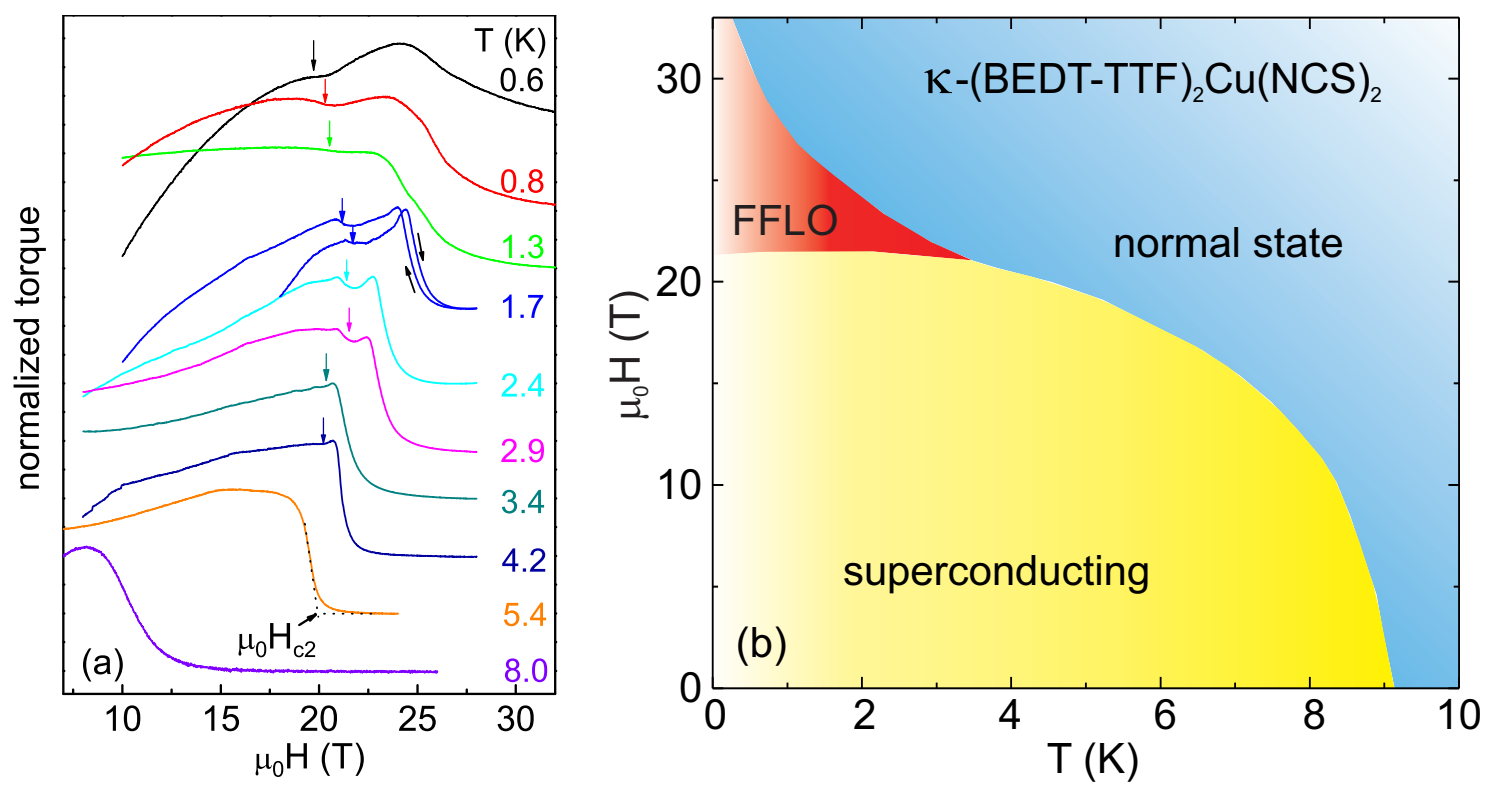

A schematic magnetic phase diagram constructed from above data is shown in Figure 5b. From the very steep initial increase of $\mu_{0} H_{c 2}$ an orbital critical field of roughly $130 \mathrm{~T}$ can be estimated for $\kappa$-(BEDT-TTF $)_{2} \mathrm{Cu}(\mathrm{NCS})_{2}$. This large value is caused by the strongly reduced orbital currents for this in-plane field orientation, as mentioned above. The steep initial slope quickly levels off at higher fields upon reaching the Pauli limit. Using $\Delta_{0} / k_{B} T_{c}=2.4$, i.e., the normalized superconducting gap as determined in earlier specific-heat studies [40], leads to $\mu_{0} H_{P}=23 \mathrm{~T}$. That fits nicely with the field of about $21 \mathrm{~T}$ where the sharp first-order phase transition emerges (Figure 4). Above this field, a strong upturn of the $\mu_{0} H_{c 2}$ line occurs. Together with this increased slope, the second phase transition appears. This is favorably in line with the expectations for the existence of an FFLO state. Indeed, the 2D organic superconductors are some of the very few materials for which this fascinating spatially inhomogeneous superconducting state can be studied.

\subsection{Nature of the Superconducting State}

For the 2D organic superconductors, the controversial experimental situation does not allow a definite conclusion as to whether conventional BCS or unconventional superconductivity exists. It is, however, well proven that the Cooper pairs are in a singlet spin state as evidenced, e.g., by NMR experiments $[41,42]$ and, as discussed above, by the Pauli limitation of the upper critical field and the existence of FFLO states.

One motivation for suggesting an unconventional order parameter of singlet $d$-wave symmetry is based on the schematic $P-T$ phase diagram of the $\kappa$-phase superconductors shown in the left panel of Figure 6 [43]. Next to the superconducting state an antiferromagnetic insulating state 
exists, resembling phase diagrams observed, e.g., for heavy-fermion and many high- $T_{c}$ materials. The one $\kappa$-phase material known that shows an antiferromagnetic insulating state at ambient pressure is $\kappa$-(BEDT-TTF $)_{2} \mathrm{Cu}\left[\mathrm{N}(\mathrm{CN})_{2}\right] \mathrm{Cl}$. A moderate pressure of 300 bar is sufficient to induce superconductivity at a record $T_{c}$ of about $12.8 \mathrm{~K}$ (Figure 6, left) [44]. A further increase in pressure rapidly suppresses superconductivity. In fact, $\kappa$-(BEDT-TTF $)_{2} \mathrm{Cu}(\mathrm{NCS})_{2}$ displays the largest pressure dependence of $T_{c}$ yet observed for any superconductor, $\Delta T_{c} / \Delta p \approx-3 \mathrm{~K} / \mathrm{kbar}[45]$.

Figure 6. (left) Schematic $P-T$ phase diagram for $\kappa$-(BEDT-TTF) $)_{2} X$ salts depicting regions of antiferromagnetic-insulator (AFM) behavior and superconductivity (SC). The arrows indicate starting points for different materials (the anion $X$ is stated) at ambient pressure. The right conceptual phase diagram after [46] for (BEDT-TTF) $)_{2} X$ salts relates the observed phases to a Mott transition. $U$ is the on-site Coulomb repulsion and $W$ denotes the electronic bandwidth.
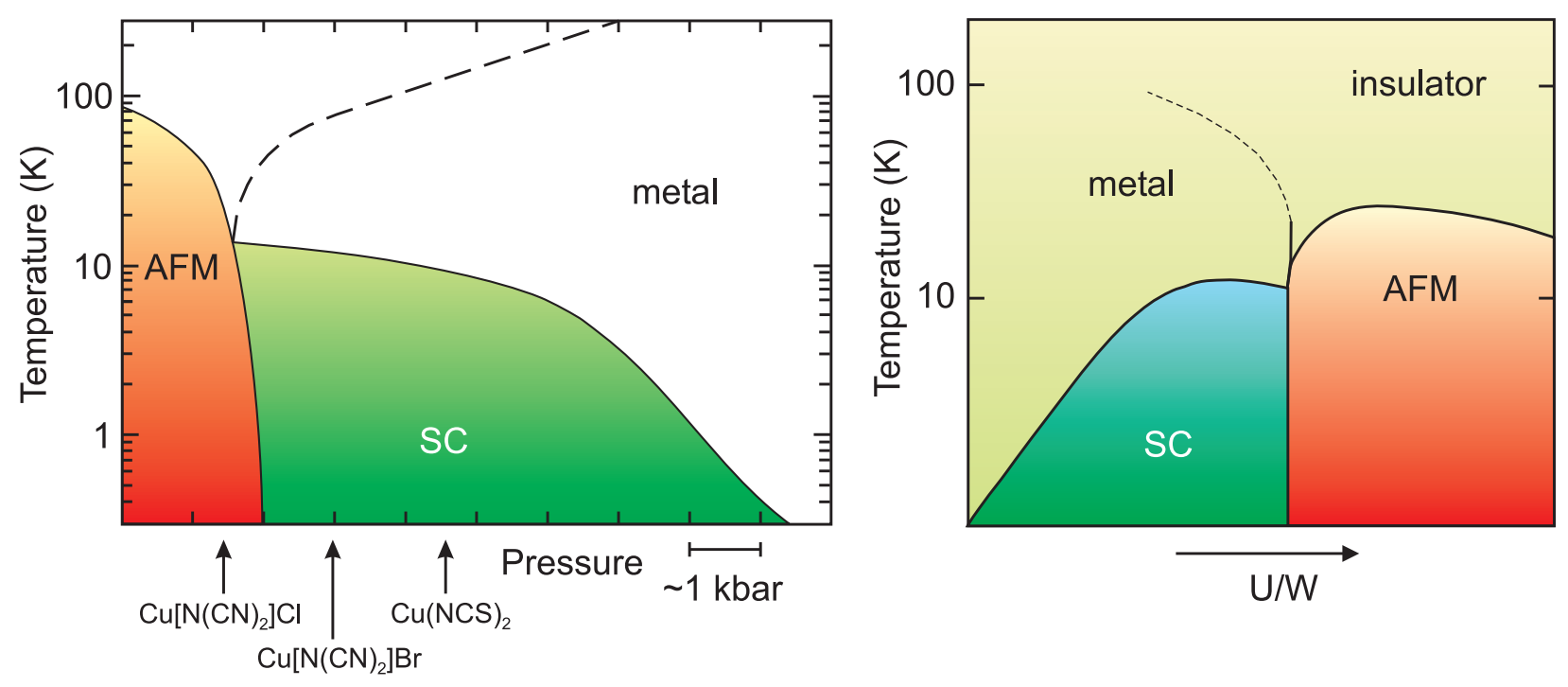

In contrast to the cuprate high- $T_{c}$ materials, the crucial parameter for organic superconductors is pressure or chemical substitution, not the charge-carrier concentration. Growing pressure increases the bandwidth $W$ of the electronic bands. Therefore, the phase diagram of the $\kappa$-phase salts might be explained by a bandwidth-controlled Mott transition as visualized by the conceptual phase diagram in the right panel of Figure 6, first proposed by Kanoda [46]. Thereby, pressure controls $U / W$, where $U$ is the on-site Coulomb repulsion. The dashed lines in the $P-T$ phase diagrams delineate different electronic-transport regimes. At high temperatures the resistivity shows a semiconducting behavior, whereas after a maximum in resistance a quadratic temperature dependence of the resistivity appears at low temperatures above $T_{c}$.

Largely unsettled is the question on the exchange interaction mediating Cooper-pair coupling. Although the proximity of the antiferromagnetic state in the $P-T$ phase diagram (Figure 6) may suggest spin fluctuations responsible for superconductivity, there are a number of experiments showing the existence of a considerable electron-phonon coupling in organic superconductors. Usually, one of the first things investigated to prove electron-phonon interaction as the pairing mechanism is the isotope effect. In the most simple BCS picture, $T_{c} \propto m^{-1 / 2}$ is expected, with $m$ being the isotope 
mass. Other mass dependences, however, are still explicable within BCS theory with a more elaborate electron-phonon coupling theory. For the $2 \mathrm{D}$ organic superconductors the experimental situation appears somewhat confusing at first sight.

As shown in Figure 7, a nonuniform $T_{c}$ shift is found for $\kappa$-phase superconductors when all eight protons in BEDT-TTF are substituted by deuterium [11]. For $\kappa$-(BEDT-TTF) $)_{2} \mathrm{Cu}(\mathrm{NCS})_{2}$ (Figure 7a), a large inverse isotope effect appears, whereas for $\kappa$-(BEDT-TTF) ${ }_{2} \mathrm{Cu}\left[\mathrm{N}(\mathrm{CN})_{2}\right] \mathrm{Br}$ (Figure $7 \mathrm{~b}$ ) an equally large "normal" isotope effect is found. The observed shifts of $4 \%-5 \%$ in $T_{c}$ are much larger than could possibly be explained by a simple molecular-mass change. The $T_{c}$ shift in $\kappa$-(BEDT-TTF) $)_{2} \mathrm{Cu}(\mathrm{NCS})_{2}$ can be rationalized by a "geometrical" effect, i.e., by internal uniaxial pressure perpendicular to the planes. Thermal-expansion measurements have shown that uniaxial pressure reduces $T_{c}$ by about $6 \mathrm{~K} / \mathrm{kbar}$ in $\kappa$-(BEDT-TTF $)_{2} \mathrm{Cu}(\mathrm{NCS})_{2}$ [47]. Consequently, the inverse isotope effect might be understood by the smaller zero-point spacing of the carbon-deuterium bonds compared to the lighter carbon-hydrogen bonds. This causes a reduced internal pressure and leads to an increased $T_{c}$ for the deuterated samples [48].

Figure 7. Magnetization curves for (a) two protonated $\left(\mathrm{h}_{8}\right)$ and two deuterated $\left(\mathrm{d}_{8}\right)$ $\kappa$-(BEDT-TTF $)_{2} \mathrm{Cu}(\mathrm{NCS})_{2}$ samples and for $(\mathbf{b})$ a protonated and deuterated $\kappa$-(BEDT$\mathrm{TTF})_{2} \mathrm{Cu}\left[\mathrm{N}(\mathrm{CN})_{2}\right] \mathrm{Br}$ sample in two different fields (0.1 Oe and $\left.1 \mathrm{Oe}\right)$ [11].

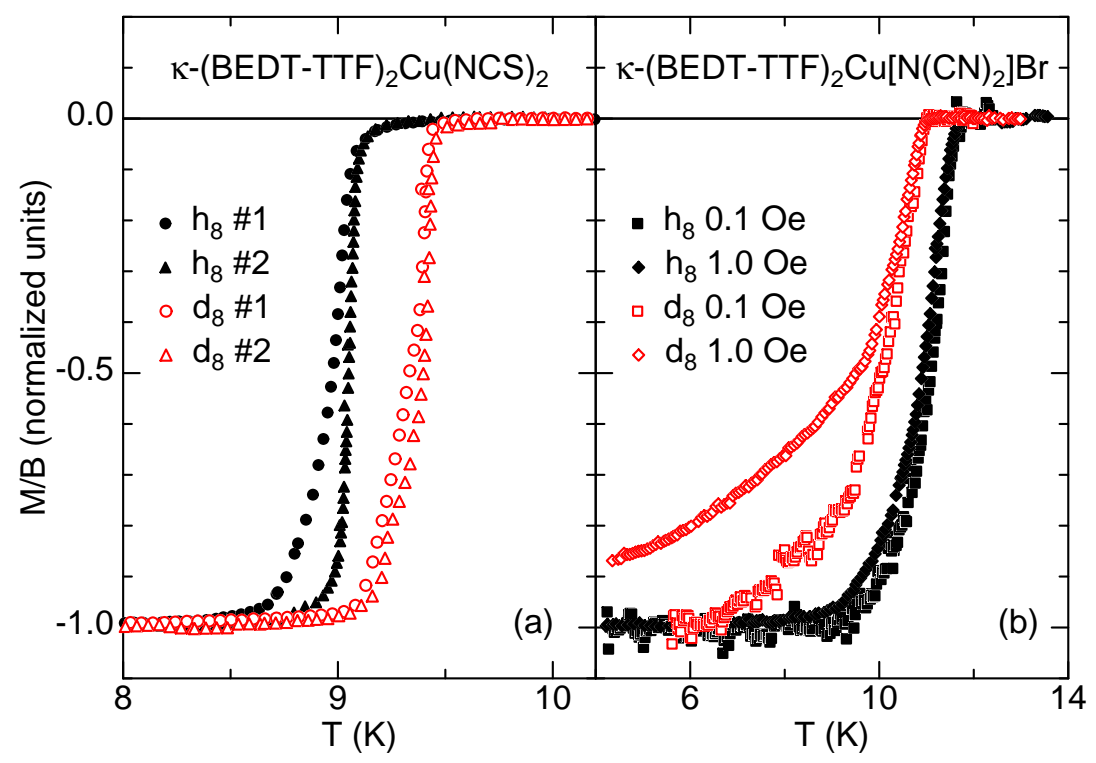

Such a scenario, however, cannot explain the $T_{c}$ reduction observed for deuterated $\kappa$-(BEDT$\mathrm{TTF})_{2} \mathrm{Cu}\left[\mathrm{N}(\mathrm{CN})_{2}\right] \mathrm{Br}$ samples (Figure $7 \mathrm{~b}$ ). But, as mentioned above, this material is situated close to an insulating state (Figure 6). Indeed, fast-cooled deuterated $\kappa$-(BEDT-TTF) ${ }_{2} \mathrm{Cu}\left[\mathrm{N}(\mathrm{CN})_{2}\right] \mathrm{Br}$ samples reflect only partial superconductivity [49]. As visible in Figure 7b, the deuterated sample shows a much higher sensitivity to applied magnetic fields. Already in $1 \mathrm{Oe}$, the transition curves becomes broad and no diamagnetic saturation is achieved. Anyway, at low enough external field ( $0.1 \mathrm{Oe})$ bulk superconductivity for the deuterated sample is found.

Investigations of the isotope effect in 2D organic superconductors were further pursued by substituting four ${ }^{12} \mathrm{C}$ atoms by ${ }^{13} \mathrm{C}$ and all eight ${ }^{32} \mathrm{~S}$ atoms by ${ }^{34} \mathrm{~S}$ in the ET molecules [50]. By that, any changes 
of the anion-hydrogen interaction were avoided. The found $T_{c}$ reduction of about $1 \%$ agrees well with the BCS expectation and provides strong support for a Cooper-pair coupling which is at least partially mediated by phonons.

Additional support for electron-phonon coupling comes from Raman data which showed a clear hardening of the Raman modes below $T_{c}$ [51] and from inelastic neutron-scattering experiments [52]. In the latter measurement a significant shift of a phonon branch was observed below $T_{c}$, an effect hardly seen this clear in any other superconductor. Summarizing, there is numerous experimental evidence for electron-phonon-mediated superconductivity, whereas the argument for a pairing mechanism originating in antiferromagnetic fluctuations is largely based on the $P-T$ phase diagram (Figure 6).

Apart from the coupling mechanism the symmetry of the order parameter is a highly controversial issue. The experimental situation still is rather inconclusive and only some aspects shall be presented here. The superconducting gap structure has been examined, e.g., by scanning tunneling spectroscopy hinting at a $d_{x^{2}-y^{2}}$ symmetry [53]. Thermal-conductivity data [54] also point to $d$-wave symmetry; this time, however, of $d_{x y}$-type symmetry. On the other hand, there exist numerous studies pointing towards a complete $s$-wave-like superconducting gap. These issues are discussed in the excellent review by Lang and Müller [5]. For further discussion of this topic see References [4,7,12-15,30].

Figure 8. Temperature dependence of the normalized spin-lattice relaxation rate. The solid line depicts the behavior for the BCS superconductor aluminum [56]. Data from ${ }^{13} \mathrm{C} \mathrm{NMR}$ for $\kappa$-(BEDT-TTF $)_{2} \mathrm{Cu}\left[\mathrm{N}(\mathrm{CN})_{2}\right] \mathrm{Br}$ in fields parallel to the conducting ET planes are shown as closed circles [41] and dashed line [42]. There is no Hebel-Slichter peak just below $T_{\mathrm{c}}$ and the relaxation rate decreases as $T^{3}$ rather then exponentially.

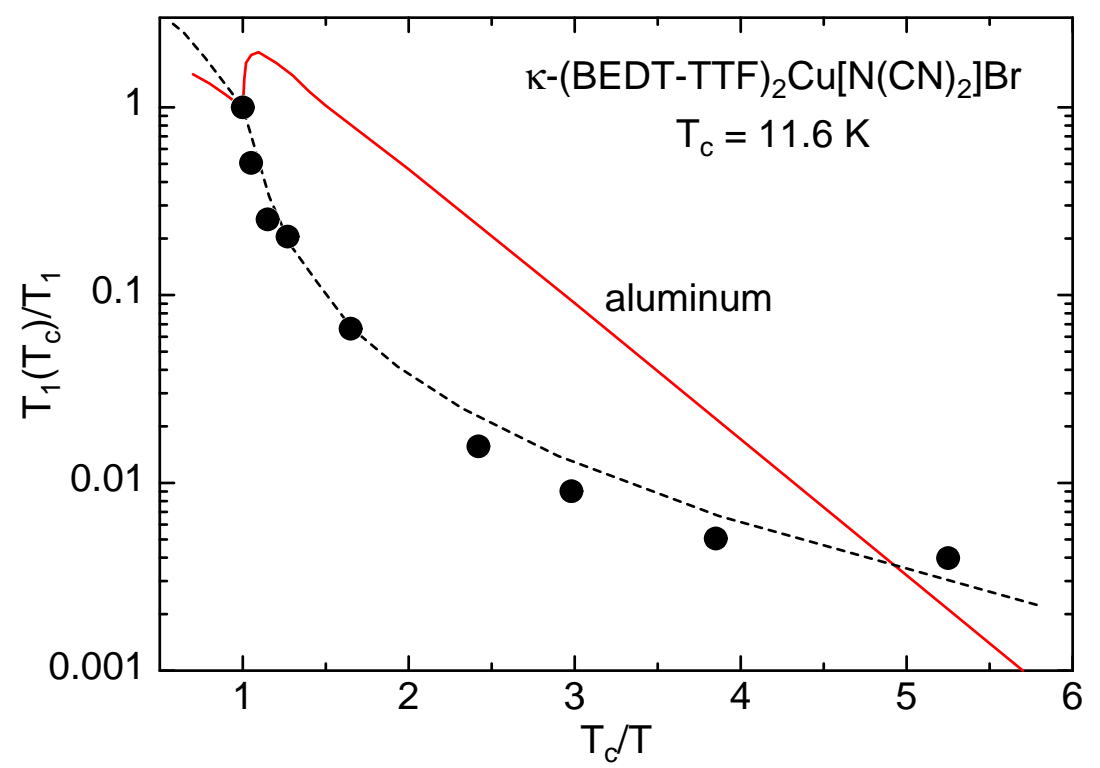

In NMR measurements of the spin-lattice relaxation rate, $1 / T_{1}$, three groups almost simultaneously reported comparable results $[41,42,55]$, two of which are shown schematically in Figure 8. Compared to the behavior found for a BCS superconductor such as aluminum (shown as the solid line in Figure 8) [56] several marked differences are observed. First, the Hebel-Slichter coherence peak, i.e., the increase of $1 / T_{1}$ just below $T_{c}$, is absent. One should note here, that although the occurrence of this peak is an 
important proof for BCS superconductivity, its absence cannot disprove conventional superconductivity as, e.g., evidenced by the missing Hebel-Slichter peak for the BCS superconductors Nb and V [56]. Second, $1 / T_{1}$ vanishes towards lower temperatures with a $T^{3}$ dependence hinting at a node-like structure of the energy gap. For a complete energy gap over the whole Fermi surface, an exponential temperature dependence, such as for aluminum, is expected. Third, the rapid decrease of $1 / T_{1}$ just below $T_{c}$ suggests a very fast opening of the gap, that is much faster than usually realized. One should note, however, that all NMR experiments were performed in applied magnetic fields, that might influence the gap structure.

Another powerful measure for deciding whether the superconducting energy gap is nodeless is the specific heat. If the electronic part of the specific heat, $C_{e}$, vanishes exponentially in the superconducting state, nodes in the energy gap can be ruled out unambiguously. A power-law in the $T$ dependence of $C_{e}$, however, indicates that line or point nodes may be present. In particular, for a $d$-wave symmetry of the gap, a $T^{2}$ behavior is expected.

Recent unpublished specific-heat data for $\beta^{\prime \prime}$-(BEDT-TTF) $)_{2} \mathrm{SF}_{5} \mathrm{CH}_{2} \mathrm{CF}_{2} \mathrm{SO}_{3}$ in zero field and with $10 \mathrm{~T}$ applied perpendicular to the ET planes are shown in Figure 9a on a double-logarithmic scale. These data favorably agree with results of an earlier experiment [57]. Due to the strong phonon contribution to the specific heat the anomaly at $T_{c}=4.3 \mathrm{~K}$ (arrow) is hardly visible in Figure 9a. In the magnetic field of $10 \mathrm{~T}$ applied along the $c$ direction, the sample is in the normal state.

Figure 9. (a) Specific heat of $\beta^{\prime \prime}$-(BEDT-TTF) $)_{2} \mathrm{SF}_{5} \mathrm{CH}_{2} \mathrm{CF}_{2} \mathrm{SO}_{3}$ as a function of temperature in a double-logarithmic scale. Data in the superconducting state in zero field and in the normal state at $10 \mathrm{~T}$ applied perpendicular to the BEDT-TTF planes are shown; (b) Temperature dependence of the specific-heat difference between the superconducting and normal state. The lines show the BCS behavior for weak coupling (dashed) [58] and strong coupling (solid) [59]. The inset shows the electronic part of the specific heat, $C_{e}$, divided by $\gamma T_{c}$ as function of $T_{c} / T$. The solid line shows the exponential vanishing of $C_{e}$ towards low $\mathrm{T}$.
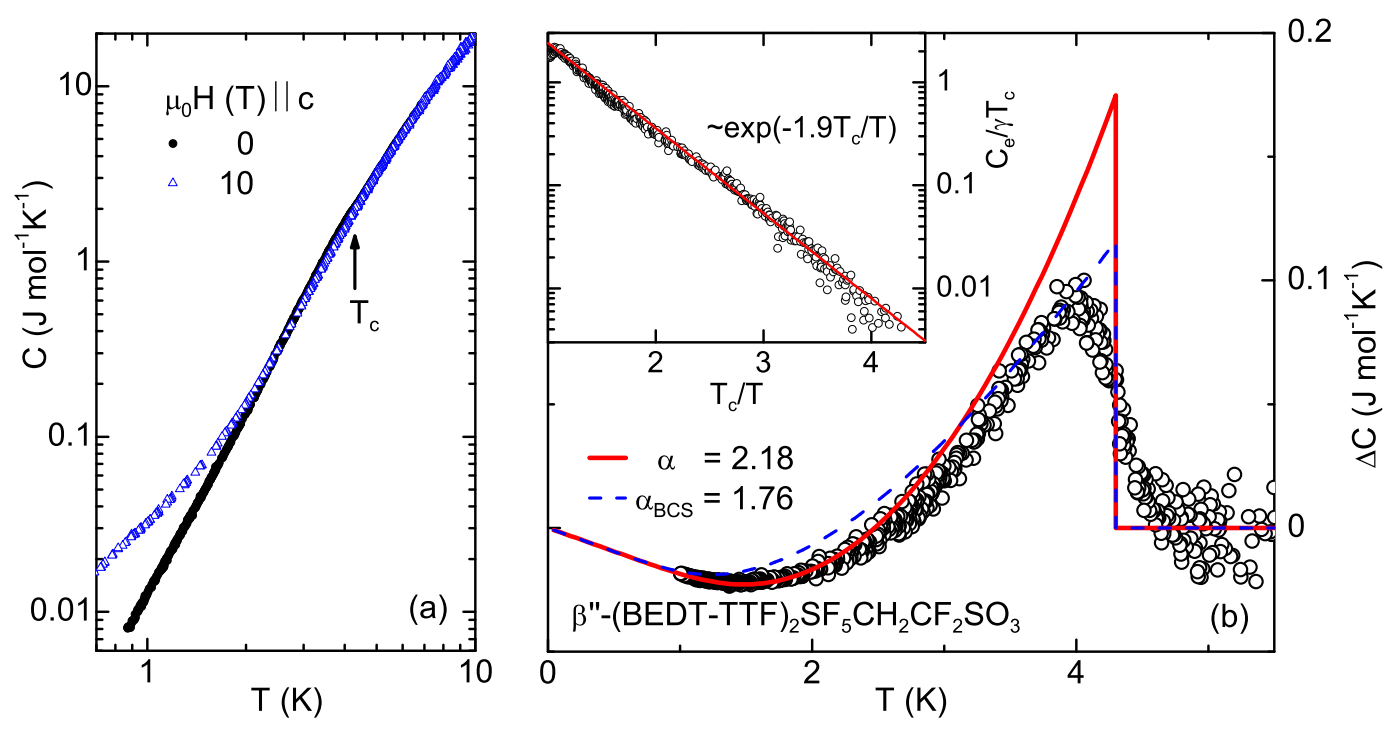

The specific-heat difference, $\Delta C$, between the superconducting $(0 \mathrm{~T})$ and normal $(10 \mathrm{~T})$ state is shown in Figure 9b. In this plot, the anomaly at $T_{c}$ now is well resolved. It is, however, rather broad 
due to fluctuation effects. The idealized specific-heat jump at $T_{c}$ and the temperature dependence of $\triangle C(T)$ does not follow the weak-coupling BCS behavior (dashed line in Figure 9b) [58]. However, the data can be well described when assuming a moderately strong coupling (solid line). Thereby, a BCS-like temperature dependence of the energy gap $\Delta C(T)$ scaled by an appropriate parameter has been assumed [59]. A reasonable description of the data is achieved for the gap ratio $\alpha=\Delta(0) / k_{B} T_{c}=2.18$, where $\alpha=1.76$ in the weak-coupling limit.

The inset of Figure 9 shows the exponential vanishing of $C_{e} \propto \exp \left(-1.9 T_{\mathrm{c}} / T\right)$ without any indication of a residual electronic contribution in the superconducting state. Such an exponential dependence has as well been found for three other organic superconductors $[40,60,61]$ in line with reports by other groups $[62,63]$. These specific-heat results, therefore, prove the existence of a complete superconducting gap and are strictly against any unconventional nodal order parameter. It is, however, fair to say, that these measurements are not sensitive to a particular direction in $k$ space and, therefore, cannot resolve any possible anisotropy of the superconducting gap.

Recently, high-resolution specific-heat measurements for two $\kappa$-phase superconductors were reported that indicated $T^{2}$ dependences of the electronic contributions at low temperatures [64]. Such a dependence would be in favor for a $d$-wave gap scenario. However, in their analysis the authors allowed for different Sommerfeld coefficients, $\gamma$, in the superconducting state than extracted from the normal-state data measured in $14 \mathrm{~T}$ [64]. In particular, for the $s$-wave description of the data for $\kappa$-(BEDT-TTF $)_{2} \mathrm{Cu}\left[\mathrm{N}(\mathrm{CN})_{2}\right] \mathrm{Br}, \gamma=20.0 \mathrm{~mJ} /\left(\mathrm{mol} \mathrm{K}^{2}\right)$ was used that is $40 \%$ smaller than the value in the normal state. This discrepancy is even larger (about 53\%) for $\kappa$-(BEDT-TTF) ${ }_{2} \mathrm{Cu}(\mathrm{NCS})_{2}$. Also for the $d$-wave description, $\gamma$ values smaller than in the normal state were used. Consequently, the analysis in Reference [64] is highly questionable and cannot be taken as evidence for unconventional pairing.

\section{Conclusions}

With this review, I introduced some selected topics of the rich physics occurring in the quasi-two-dimensional organic metals. Thereby, I laid emphasis on the superconducting properties of these highly anisotropic materials. With their simple electronic structure and the easily accessible complete superconducting phase diagram these charge-transfer salts serve as model systems for 2D superconductors. The observation of the long-time predicted FFLO state allows for a better understanding of superconductivity in general, not only at high magnetic fields. The question on the nature of the superconducting state is still unsettled for the $2 \mathrm{D}$ organic metals. Controversial experimental results prevent an unequivocal assignment of the order-parameter symmetry and the coupling mechanism of the Cooper pairs. Some data suggest rather an unconventional $d$-wave-like behavior, whereas other experiments-especially specific-heat results-explicitly prove the existence of a nodeless superconducting energy gap. Further studies, particular experiments that are able to directly probe phase differences of the superconducting wave function, are highly desirable. 


\section{Acknowledgements}

I would like to thank all my coworkers with which I had the luck and honor to cooperate on organic superconductivity over the years, especially the collaborators of the recent years: B. Bergk, R. Beyer, R. Lortz, J.A. Schlueter, and G. Zwicknagl.

\section{References}

1. Jérome, D.; Mazaud, A.; Ribault, M.; Bechgaard, K. Superconductivity in a synthetic organic conductor (TMTSF) ${ }_{2} \mathrm{PF}_{6}$. J. Phys. Lett. 1980, 41, L95-L98.

2. Ishiguro, T.; Yamaji, K.; Saito, G. Organic Superconductors; Springer: Berlin, Heidelberg/Germany, 1998.

3. Jérome, D. Organic conductors: From charge density wave TTF-TCNQ to superconducting (TMTSF) ${ }_{2} \mathrm{PF}_{6}$. Chem. Rev. 2004, 104, 5565-5591.

4. Maple, M.B.; Bauer, E.D.; Zapf, V.S.; Wosnitza, J. Unconventional Superconductivity in Novel Materials. In Superconductivity; Bennemann, K.H., Ketterson, J.B., Eds.; Springer: Berlin/ Heidelberg, Germany, 2008; Volume I, pp. 639-762.

5. Lang, M.; Müller, J. Organic Superconductors. In Superconductivity; Bennemann, K.H., Ketterson, J.B., Eds.; Springer: Berlin/Heidelberg, Germany, 2008; Volume II, pp. 1155-1223.

6. Kobayashi, H.; Cui, H.; Kobayashi, A. Organic metals and superconductors based on BETS (BETS = Bis(ethylenedithio)tetraselenafulvalene). Chem. Rev. 2004, 104, 5265-5288.

7. Lang, M. Quasi-two-dimensional organic superconductors. Supercond. Rev. 1996, 2, 1-115.

8. McKenzie, R.H. Similarities between organic and cuprate superconductors. Science 1997, 278, 820-821.

9. McKenzie, R.H. A strongly correlated electron model for the layered organic superconductors $\kappa$-(BEDT-TTF $)_{2}$ X. Comments Cond. Mat. Phys. 1998, 18, 309-337.

10. Wosnitza, J. Superconducting properties of quasi-two-dimensional organic metals. Physica C 1999, 317-318, 98-107.

11. Wosnitza, J. Perspectives on the nature of superconductivity in organic metals. J. Low Temp. Phys. 1999, 117, 1701-1710.

12. Wosnitza, J. Superconductivity in Low-Dimensional Organic Metals. In Studies of High-Temperature Superconductivity; Narlikar, A.V., Ed.; Nova Science Publishers: Huntington, VA, USA, 2000; Volume 34, pp. 97-131.

13. Wosnitza, J. Recent aspects of organic metals and superconductors. Curr. Opin. Solid State Mater. Sci. 2001, 5, 131-141.

14. Singleton, J.; Mielke, C. Quasi-two-dimensional organic superconductors: A review. Contemp. Phys. 2002, 43, 63-96.

15. Wosnitza, J. Quasi-two-dimensional organic superconductors. J. Low Temp. Phys. 2007, 146, 641-667.

16. Williams, J.M.; Ferraro, J.R.; Thorn, R.J.; Carlson, K.D.; Geiser, U.; Wang, H.H.; Kini, A.M.; Whangbo, M.H. Organic Superconductors: Synthesis, Structure, Properties, and Theory; Prentice Hall: Englewood Cliffs, NJ, USA, 1992. 
17. Wosnitza, J. Fermi Surfaces of Low-Dimensional Organic Metals and Superconductors; Springer: Berlin/Heidelberg, Germany, 1996.

18. Mori, T.; Kobayashi, A.; Sasaki, Y.; Kobayashi, H.; Saito, G.; Inokuchi, H. Band structures of two types of (BEDT-TTF) $\mathrm{I}_{3}$. Chem. Lett. 1984, 6, 957-960.

19. Kartsovnik, M.V. High magnetic fields: A tool for studying electronic properties of layered organic metals. Chem. Rev. 2004, 104, 5737-5782.

20. Kartsovnik, M.V.; Laukhin, V.N.; Pesotskii, S.I.; Schegolev, I.F.; Yakovenko, V.M. Angular magnetoresistance oscillations and the shape of the Fermi surface in $\beta-\left(\mathrm{ET}_{2} \mathrm{IBr}_{2}\right.$. J. Phys. I France 1992, 2, 89-99.

21. Kobayashi, A.; Kato, R.; Kobayashi, H.; Moriyama, S.; Nishio, Y.; Kajita, K.; Sasaki, W. Crystal and electronic structures of a new molecular superconductor, $\kappa$-(BEDT-TTF $)_{2} \mathrm{I}_{3}$. Chem. Lett. 1987, $6,459-462$.

22. Oshima, K.; Mori, T.; Inokuchi, H.; Urayama, H.; Yamochi, H.; Saito, G. Shubnikov-de Haas effect and the Fermi surface in an ambient-pressure organic superconductor [bis(ethylenedithiolo)tetrathiafulvalene $]_{2} \mathrm{Cu}(\mathrm{NCS})_{2}$. Phys. Rev. B 1988, 38, 938-941.

23. Mori, H.; Tanaka, S.; Oshima, M.; Saito, G.; Mori, T.; Murayama, Y.; Inokuchi, H. Crystal and electronic structures of (BEDT-TTF $)_{2}\left[\mathrm{MHg}(\mathrm{SCN})_{4}\right]\left(\mathrm{M}=\mathrm{K}\right.$ and $\left.\mathrm{NH}_{4}\right)$. Bull. Chem. Soc. Jpn. 1990, 63, 2183-2190.

24. Kartsovnik, M.V.; Andres, D.; Biberacher, W. Hierarchy of the density-wave states and superconductivity in the organic conductor $\alpha$-(BEDT-TTF $)_{2} \mathrm{KHg}(\mathrm{SCN})_{4}$. C. R. Chim. 2007, 10, 6-14.

25. Beckmann, D.; Wanka, S.; Wosnitza, J.; Schlueter, J.A.; Williams, J.M.; Nixon, P.G.; Winter, R.W.; Gard, G.L.; Ren, J.; Whangbo, M.-H. Characterization of the Fermi surface of the organic superconductor $\beta^{\prime \prime}-(\mathrm{ET})_{2} \mathrm{SF}_{5} \mathrm{CH}_{2} \mathrm{CF}_{2} \mathrm{SO}_{3}$ by measurements of Shubnikov-de Haas and angle-dependent magnetoresistance oscillations and by electronic band-structure calculations. Eur. Phys. J. B 1998, 1, 295-300.

26. Chandrasekhar, B.S. A note on the maximum critical field of high-field superconductors. Appl. Phys. Lett. 1962, 1, 7-8.

27. Clogston, A.M. Upper limit for the critical field in hard superconductors. Phys. Rev. Lett. 1962, 9 , 266-267.

28. Lang, M.; Steglich, F.; Toyota, N.; Sasaki, T. Fluctuation effects and mixed-state properties of the layered organic superconductors $\kappa$-(BEDT-TTF $)_{2} \mathrm{Cu}(\mathrm{NCS})_{2}$ and $\kappa$-(BEDT-TTF $)_{2} \mathrm{Cu}\left[\mathrm{N}(\mathrm{CN})_{2}\right] \mathrm{Br}$. Phys. Rev. B 1994, 49, 15227-15234.

29. Hagel, J.; Wanka, S.; Beckmann, D.; Wosnitza, J.; Schweitzer, D.; Strunz, W.; Thumfart, M. Vortex dynamics of the organic superconductor $\kappa-(\mathrm{ET})_{2} \mathrm{Cu}\left[\mathrm{N}(\mathrm{CN})_{2}\right]$ Br. Physica $C$ 1997, 291, 213-222.

30. Wosnitza, J. Superconducting properties of quasi-two-dimensional organic metals. Physica C 1999, 317-318, 98-107.

31. Wanka, S.; Beckmann, D.; Wosnitza, J.; Balthes, E.; Schweitzer, D.; Strunz, W.; Keller, H.J. Critical fields and mixed-state properties of the layered organic superconductor $\kappa$-(BEDT-TTF $)_{2} \mathrm{I}_{3}$. Phys. Rev. B 1996, 49, 9301-9309. 
32. Müller, P. Intrinsic Josephson Effects in Layered Superconductors. In Advances in Solid State Physics; Helbig, R., Ed.; Vieweg: Braunschweig, Germany, 1994; Volume 34, pp. 1-33.

33. Fulde, P.; Ferrell, R.A. Superconductivity in a strong spin exchange field. Phys. Rev. 1964, 135, A550-A563.

34. Larkin, A.I.; Ovchinnikov, Y.N. Inhomogeneous state of superconductors. Zh. Eksp. Teor. Fiz. 1964, 47, 1136-1146; Sov. Phys. JETP 1965, 20, 762-768.

35. Maki, K.; Tsuneto, T. Pauli paramagnetism and superconducting state. Prog. Theor. Phys. 1964, $31,945-956$.

36. Gruenberg, L.W.; Gunther, L. Fulde-Ferrell effect in type-II superconductors. Phys. Rev. Lett. 1966, 16, 996-998.

37. Zwicknagl, G.; Wosnitza, J. Breaking Translational Invariance by Population Imbalance: The Fulde-Ferrell-Larkin-Ovchinnikov States. In BCS: 50 years; Cooper, L.N., Feldman, D., Eds.; World Scientific: Singapore, 2010; pp. 337-371.

38. Lortz, R.; Wang, Y.; Demuer, A.; Böttger, P.H.M.; Bergk, B.; Zwicknagl, G.; Nakazawa, Y.; Wosnitza, J. Calorimetric evidence for a Fulde-Ferrell-Larkin-Ovchinnikov superconducting state in the layered organic superconductor $\kappa$-(BEDT-TTF $)_{2} \mathrm{Cu}(\mathrm{NCS})_{2}$. Phys. Rev. Lett. 2007, 99, 187002:1-187002:4.

39. Bergk, B.; Demuer, A.; Sheikin, I.; Wang, Y.; Wosnitza, J.; Nakazawa, Y.; Lortz, R. Magnetic torque evidence for the Fulde-Ferrell-Larkin-Ovchinnikov state in the layered organic superconductor $\kappa$-(BEDT-TTF $)_{2} \mathrm{Cu}(\mathrm{NCS})_{2}$. Phys. Rev. B 2011, 83, 064506:1-064506:7.

40. Wosnitza, J.; Wanka, S.; Hagel, J.; Reibelt, M.; Schweitzer, D.; Schlueter, J.A. Thermodynamic properties of quasi-two-dimensional organic superconductors. Synth. Met. 2003, 133-134, 201-203.

41. Soto, S.M.D.; Slichter, C.P.; Kini, A.M.; Wang, H.H.; Geiser, U.; Williams, J.M. ${ }^{13}$ C NMR studies of the normal and superconducting states of the organic superconductor $\kappa-(\mathrm{ET})_{2} \mathrm{Cu}\left[\mathrm{N}(\mathrm{CN})_{2}\right] \mathrm{Br}$. Phys. Rev. B 1995, 52, 10364-10368.

42. Mayaffre, H.; Wzietek, P.; Jérome, D.; Lenoir, C.; Batail, P. Superconducting state of $\kappa$ - $(\mathrm{ET})_{2} \mathrm{Cu}\left[\mathrm{N}(\mathrm{CN})_{2}\right] \mathrm{Br}$ studied by ${ }^{13} \mathrm{C} \mathrm{NMR}$ : Evidence for vortex-core-induced nuclear relaxation and unconventional pairing. Phys. Rev. Lett. 1995, 75, 4122-4125.

43. Wzietek, P.; Mayaffre, H.; Jérome, D.; Brazovskii, S. NMR in the 2D organic superconductors. J. Phys. I France 1996, 6, 2011-2041.

44. Williams, J.M.; Kini, A.M.; Wang, H.H.; Carlson, K.D.; Geiser, U.; Montgomery, L.K.; Pyrka, G.J.; Watkins, D.M.; Kommers, J.M.; Boryschuk, S.J.; et al. From semiconductorsemiconductor transition $(42 \mathrm{~K})$ to the highest- $T_{c}$ organic superconductor, $\kappa-(\mathrm{ET}) 2 \mathrm{Cu}[\mathrm{N}(\mathrm{CN}) 2] \mathrm{Cl}$ $\left(T_{c}=12.5 \mathrm{~K}\right)$. Inorg. Chem. 1990, 29, 3272-3274.

45. Sadewasser, S.; Looney, C.; Schilling, J.S.; Schlueter, J.A.; Williams, J.M.; Nixon, P.G.; Winter, R.W.; Gard, G.L. Dependence of $T_{c}$ on hydrostatic pressure in $\beta^{\prime \prime}-(\mathrm{ET})_{2} \mathrm{SF}_{5} \mathrm{CH}_{2} \mathrm{CF}_{2} \mathrm{SO}_{3}$ and $\kappa-(\mathrm{ET})_{2} \mathrm{Cu}(\mathrm{NCS})_{2}$. Solid State Commun. 1997, 104, 571-575.

46. Kanoda, K. Recent progress in NMR studies on organic conductors. Hyperfine Interact. 1997, 104, 235-249. 
47. Müller, J.; Lang, M.; Steglich, F.; Schlueter, J.A.; Kini, A.M. Geiser, U.; Mohtasham, J.; Winter, R.W.; Gard, G.L.; Sasaki, T., et al. Comparative thermal-expansion study of $\beta^{\prime \prime}-(\mathrm{ET})_{2} \mathrm{SF}_{5} \mathrm{CH}_{2} \mathrm{CF}_{2} \mathrm{SO}_{3}$ and $\kappa-(\mathrm{ET})_{2} \mathrm{Cu}(\mathrm{NCS})_{2}$ : Uniaxial pressure coefficients of $T_{c}$ and upper critical fields. Phys. Rev. B 2000, 61, 11739-11734.

48. Kini, A.M.; Schlueter, J.A.; Ward, B.H.; Geiser, U.; Wang, H.H. On the "inverse" isotope effect in organic superconductors: New findings and implications. Synth. Met. 2001, 120, 713-714.

49. Kawamoto, A.; Miyagawa, K.; Kanoda, K. Deuterated $\kappa$-(BEDT-TTF $)_{2} \mathrm{Cu}\left[\mathrm{N}(\mathrm{CN})_{2}\right] \mathrm{Br}$ : A system on the border of the superconductor-magnetic-insulator transition. Phys. Rev. B 1997, 55, 14140-14143.

50. Kini, A.M.; Carlson, K.D.; Wang, H.H.; Schlueter, J.A.; Dudek, J.D.; Sirchio, S.A.; Geiser, U.; Lykke, K.R.; Williams, J.M. Determination of a mass isotope effect on $T_{c}$ in an electron-donor-based organic superconductor, $\kappa$-(ET) ${ }_{2} \mathrm{Cu}(\mathrm{NCS})_{2}$, where ET represents bis(ethylenedithio)tetrathiafulvalene. Physica C 1996, 264, 81-94.

51. Pedron, D.; Visentini, G.; Bozio, R.; Williams, J.M.; Schlueter, J.A. Phonon dynamics and superconductivity in the organic crystal $\kappa$-(BEDT-TTF $)_{2} \mathrm{Cu}\left[\mathrm{N}(\mathrm{CN})_{2}\right] \mathrm{Br}$. Physica $C$ 1997, 276, $1-8$.

52. Pintschovius, L.; Rietschel, H.; Sasaki, T.; Mori, H.; Tanaka, S.; Toyota, N.; Lang, M.; Steglich, F. Observation of superconductivity-induced phonon frequency changes in the organic superconductor $\kappa$-(BEDT-TTF $)_{2} \mathrm{Cu}(\mathrm{NCS})_{2}$. Europhys. Lett. 1997, 37, 627-632.

53. Arai, T.; Ichimura, K.; Nomura, K.; Takasaki, S.; Yamada, J.; Nakatsuji, S.; Anzai, H. Tunneling spectroscopy on the organic superconductor $\kappa$-(BEDT-TTF $)_{2} \mathrm{Cu}(\mathrm{NCS})_{2}$ using STM. Phys. Rev. $B$ 2001, 63, 104518:1-104518:5.

54. Izawa, K.; Yamaguchi, H.; Sasaki, T., Matsuda, Y. Superconducting gap structure of $\kappa$-(BEDT$\mathrm{TTF})_{2} \mathrm{Cu}(\mathrm{NCS})_{2}$ probed by thermal conductivity tensor. Phys. Rev. Lett. 2002, 88, 027002:1-027002:4.

55. Kanoda, K.; Miyagawa, K.; Kawamoto, A.; Nakazawa, Y. NMR relaxation rate in the superconducting state of the organic conductor $\kappa$-(BEDT-TTF $)_{2} \mathrm{Cu}\left[\mathrm{N}(\mathrm{CN})_{2}\right] \mathrm{Br}$. Phys. Rev. B 1996, $54,76-79$.

56. Ginsberg, D.M.; Hebel, L.C. The Green's Function Method. In Superconductivity; Parks, R., Ed.; Marcel Dekker: New York, NY, USA, 1969; Volume 1, pp. 193-258.

57. Wanka, S.; Hagel, J.; Beckmann, D.; Wosnitza, J.; Schlueter, J.A.; Williams, J.M.; Nixon, P.G.; Winter, R.W.; Gard, G.L. Specific heat and critical fields of the organic superconductor $\beta^{\prime \prime}$-(BEDT-TTF) $)_{2} \mathrm{SF}_{5} \mathrm{CH}_{2} \mathrm{CF}_{2} \mathrm{SO}_{3}$. Phys. Rev. B 1998, 57, 3084-3088.

58. Mühlschlegel, B. Die thermodynamischen Funktionen des Supraleiters. Z. Phys. 1959, 155, 313-327.

59. Padamsee, H.; Neighbor, J.E.; Schiffman, C.A. Quasiparticle phenomenology for thermodynamics of strong-coupling superconductors. J. Low Temp. Phys. 1973, 12, 387-411.

60. Wosnitza, J.; Liu, X.; Schweitzer, D.; Keller, H.J. Specific heat of the organic superconductor $\kappa$-(BEDT-TTF) ${ }_{2} \mathrm{I}_{3}$. Phys. Rev. B 1994, 50, 12747-12751. 
61. Elsinger, H.; Wosnitza, J.; Wanka, S.; Hagel, J.; Schweitzer, D.; Strunz, W. $\kappa$-(BEDT$\mathrm{TTF})_{2} \mathrm{Cu}\left[\mathrm{N}(\mathrm{CN})_{2}\right] \mathrm{Br}$ : A fully gapped strong-coupling superconductor. Phys. Rev. Lett. 2000, 84, 6098-6101.

62. Kovalev, A.E.; Ishiguro, T.; Yamada, J.; Takasaki, S.; Anzai, H. The anisotropic low-temperature specific heat of organic superconductor $\kappa$-(BEDT-TTF $)_{2} \mathrm{Cu}(\mathrm{NCS})_{2}$ in magnetic field. J. Exp. Theor. Phys. 2001, 92, 1035-1037.

63. Müller, J.; Lang, M.; Helfrich, R.; Steglich, F.; Sasaki, T. High-resolution ac-calorimetry studies of the quasi-two-dimensional organic superconductor $\kappa$-(BEDT-TTF $)_{2} \mathrm{Cu}(\mathrm{NCS})_{2}$. Phys. Rev. B 2002, 65, 140509:1-140509:4.

64. Taylor, O.J.; Carrington, A.; Schlueter, J.A. Specific-Heat Measurements of the Gap Structure of the Organic Superconductors $\kappa-(\mathrm{ET})_{2} \mathrm{Cu}\left[\mathrm{N}(\mathrm{CN})_{2}\right] \mathrm{Br}$ and $\kappa-(\mathrm{ET})_{2} \mathrm{Cu}(\mathrm{NCS})_{2}$. Phys. Rev. Lett. 2007, 99, 057001:1-057001:4.

(c) 2012 by the author; licensee MDPI, Basel, Switzerland. This article is an open access article distributed under the terms and conditions of the Creative Commons Attribution license (http://creativecommons.org/licenses/by/3.0/.) 